\title{
Continuous Dependence of the Singular Nonlinear Van der Pol Equation Solutions with Respect to the Boundary Conditions: Elements of $p$-regularity Theory
}

\author{
Beata Medak ${ }^{1}$ (D) Alexey A. Tret'yakov ${ }^{1,2,3}$ (D)
}

Received: 10 November 2019 / Revised: 12 March 2020 / Published online: 9 May 2020

(c) The Author(s) 2020

\begin{abstract}
This paper studies the problem of the continuous dependence of Van der Pol equation solutions with respect to the boundary conditions. We provide a new approach for the existence of such solutions via $p$-regularity theory. Several existence theorems about continuous solutions are established.
\end{abstract}

Keywords $p$-regularity $\cdot p$-factor operator $\cdot$ Nonlinear boundary value problems $\cdot$ Implicit function theorem · Van der Pol equation

Mathematics Subject Classification Primary 34B15 · 34B16 · 58C15; Secondary 47J05

\section{Introduction}

The continuous dependence of the solutions of differential equations with respect to the boundary conditions is a difficult topic in mathematical sciences. Usually such a problem is given as a nonlinear equation $F(x)=0$, where $F$ is a sufficiently smooth map between Banach spaces $X$ and $Y$. The mapping $F$ is regular at the point $x^{*}$, if the operator $F^{\prime}\left(x^{*}\right)$ is invertible, i.e. when $F^{\prime}\left(x^{*}\right)$ is surjective. The $p$-regularity theory (see, for example $[4,5,11]$ ) deals with nonregular (degenerate, singular) cases, i.e. with the problems, when the operator $F^{\prime}\left(x^{*}\right)$ is not surjective.

Beata Medak

bmedak@uph.edu.pl

Alexey A. Tret'yakov

tret@uph.edu.pl

1 Faculty of Exact and Natural Sciences, Siedlce University of Natural Sciences and Humanities, 3-go Maja 54, 08-110 Siedlce, Poland

2 System Research Institute, Polish Academy of Sciences, Newelska 6, 01-447 Warsaw, Poland

3 Dorodnicyn Computing Center, FRS CSC RAS, Vavilova 40, Moscow, Russia 119991 
In this paper, we consider a nonlinear boundary value problem of the following type:

$$
F_{\sigma}=\ddot{x}+\sigma\left(x^{2}-1\right) \dot{x}+x+x^{p}=0, \quad x(0)=v, \quad x(2 \pi)=\rho,
$$

where $\mu=(\nu, \rho)$ are small parameters and $\mu^{*}=(0,0)$. If $\nu=0$ and $\rho=0$, then this problem is the well-known nonlinear Van der Pol equation from the theory of oscillations, and its solutions represent essential interest in different fields of physics and mathematics. This problem always has the trivial solution $x^{*}(t)=0$.

We will consider the singular case of this equation, namely, with the singular parameter $\sigma=0$ or $\sigma= \pm \sqrt{3}$, since only for these $\sigma$ the operator $F_{\sigma}^{\prime}\left(x^{*}\right)$ is singular. For simplicity, further all our constructions and investigations will be carried for $\sigma=0$, i.e. for the equation

$$
\ddot{x}+x+x^{p}=0, \quad x(0)=0, \quad x(2 \pi)=0,
$$

because the case $\sigma= \pm \sqrt{3}$ can be studied similarly. We will solve the problem of the existing continuous solution of the perturbed Van der Pol boundary value problem

$$
\ddot{x}+x+x^{p}=0, \quad x(0)=v, \quad x(2 \pi)=\rho,
$$

where $\nu$ and $\rho$ are small parameters, which we denote as $\mu(\nu, \rho)$. We consider the case $p=2$, although the case of $p>2$ is treated similarly, and based on $p$-regularity theory, we prove the existence of continuous dependent solutions of (1.3) with respect to boundary conditions $\mu$ (of course, small parameter $\mu$ ) and, more importantly, provide the formula for these solutions.

In degenerate problems, it is typical to study the existence of solutions. Usually, there is not just one solution but many solutions. This is an important problem. However, the question about the existence of continuous solutions is equally interesting because there are no results in this area. Note that in the regular case, the classical Implicit Function Theorem is often used to address such problems. In the nonregular case, this theorem cannot be applied. The results obtained in the paper are based on the generalization of the Implicit Function Theorem for nonregular mappings, which were presented, for example, in $[1,2,9,10]$. We will provide a strong definition of the constructions of $p$-regularity theory and adapt it to the issue under consideration.

Here we'll make a note about multivalued operators.

Let $X$ and $Y$ be the Banach spaces. By the mapping $\Phi: X \rightarrow 2^{Y}$ we mean a multivalued mapping (multimapping) from $X$ to the set of all subsets of a space Y. Let $\rho(x, y)=\|x-y\|$ be the distance between elements $x$ and $y$ in a Banach space and let $\rho(x, M)=\inf \{\|x-z\|$ : $z \in M\}$ be the distance from element $x$ to subset $M$ in this space. By $\operatorname{dist}_{H}\left(M_{1}, M_{2}\right)=$ $\max \left\{\sup \left\{\rho\left(x, M_{2}\right): x \in M_{1}\right\}, \sup \left\{\rho\left(x, M_{1}\right): x \in M_{2}\right\}\right\}$ we denote the Hausdorff distance between sets $M_{1}$ and $M_{2}$.

For a linear surjective operator $\Lambda: X \rightarrow Y$ we denote by $\Lambda^{-1}$ its right inverse, that is $\Lambda^{-1}: Y \rightarrow 2^{X}$ which takes an element $y \in Y$ to its complete inverse image of the mapping $\Lambda, \Lambda^{-1} y=\{x \in X: \Lambda x=y\}$, and of course $\Lambda \Lambda^{-1}=I_{Y}$. We set $\left\|\Lambda^{-1} y\right\|=\inf \{\|x\|:$ $\left.x \in \Lambda^{-1} y\right\}$. By the "norm" of such right inverse operator we mean the number

$$
\left\|\Lambda^{-1}\right\|=\sup _{\|y\|=1}\left\|\Lambda^{-1} y\right\| .
$$

By the Banach theorem on surjective linear operator, we have $\left\|\Lambda^{-1}\right\|<\infty$. Note, that if $\Lambda$ is one-to-one, then $\left\|\Lambda^{-1}\right\|$ is the usual norm of the operator $\Lambda^{-1}$. In our considerations, by $\Lambda^{-1}$ we shall mean just right inverse multivalued operator with the norm defined by (1.4).

An important element of our study is Theorem 3.1. It is an analogue of the Lyusternik Theorem on the tangent cone, which concerns the existence of continuous solutions of the 
equation $F(x, \mu)=0$, where $F: X \times M \rightarrow Z, M$ is finite dimensional space, $X, Z$ are Banach spaces and $F(x, \mu) \in C^{p+1}(X \times M)$.

In its proof, we apply the Michael Selection Theorem (see [8]), which we provide in modified form:

Theorem 1.1 Let $X, Y-B$-spaces, $A \in L(X, Y), A$ is surjective and $\left\|A^{-1}\right\| \leq K$. Then, there exists a continuous mapping $M: Y \rightarrow X$, such that $A M(y)=y$ and $\|M(y)\| \leq c\|y\|$, where $c>0$ is a constant independent of $y$.

To the end of our article we formulate two other existence theorems about continuous solutions. We can prove them analogously.

The illustration of our problem is the following singular Van der Pol nonlinear boundary value problem (1.3)

$$
F(x)=\ddot{x}+x+x^{p}=0, \quad x(0)=v, \quad x(2 \pi)=\rho,
$$

where $v$ and $\rho$ are small parameters from $U\left(v^{*}, \rho^{*}\right)=U(0,0), v^{*}=0, \rho^{*}=0$.

We point out that (1.5) always has a trivial solution $x^{*}(t)=0$ if $\mu=0$. We will consider the case $p=2$ and parameters $\mu=(\nu, \rho)$. Based on $p$-regularity theory and our theorems, in this paper, we prove that a continuous solution of Eq. (1.5), dependent on parameter $\mu$, exists for all $\mu$ sufficiently small.

\section{P-factor Lyusternik Theorem and some Elements of $p$-regularity Theory}

The apparatus of $p$-regularity is an important tool for studying nonlinear problems. In this section, we present some definitions, notations and theorems of $p$-regularity theory to be used in what follows (see [4-7,10,11]).

We are interested in the following nonlinear problem:

$$
F(x, \mu)=0,
$$

where the mapping $F: X \times M \rightarrow Z$ and $X, M$ and $Z$ are Banach spaces.

Assume that for some point $\left(x^{*}, \mu^{*}\right) \in X \times M, \operatorname{Im} F^{\prime}\left(x^{*}, \mu^{*}\right) \neq Z$. Let

$$
Z=Z_{1} \oplus \cdots \oplus Z_{p},
$$

where $Z_{1}=\operatorname{cl}\left(\operatorname{Im} F^{\prime}\left(x^{*}, \mu^{*}\right)\right)$ and $W_{1}=Z$. For $W_{2}$, we use one of the closed complement of $Z_{1}$ in $Z$ (if such one there exists). Let $P_{W_{2}}: Z \rightarrow W_{2}$ be the projector onto $W_{2}$ along $Z_{1}$. By $Z_{2}$, we denote the closure of the linear span of the image of the quadratic mapping $P_{W_{2}} F^{\prime \prime}\left(x^{*}, \mu^{*}\right)[\cdot]^{2}$. Then, inductively,

$$
Z_{i}=\operatorname{cl}\left(\operatorname{spanIm} P_{W_{i}} F^{(i)}\left(x^{*}, \mu^{*}\right)[\cdot]^{i}\right) \subseteq W_{i}, i=2, \ldots, p-1,
$$

where $W_{i}$ is a choice of closed complement of $Z_{1} \oplus \cdots \oplus Z_{i-1}, i=2, \ldots, p$ with respect to $Z$, and $P_{W_{i}}: Z \rightarrow W_{i}$ is a projector onto $W_{i}$ along $Z_{1} \oplus \cdots \oplus Z_{i-1}, i=2, \ldots, p$ with respect to $Z$. Finally, $Z_{p}=W_{p}$. The order $p$ is the minimal number (if it exists) for which the decomposition (2.2) holds. 
In what follows, we will be denote $\varphi^{(0)}=\varphi$ for any mapping $\varphi$.

Define the following mappings:

$$
f_{i}: U \subset X \times M \rightarrow Z_{i}, f_{i}(x, \mu)=P_{Z_{i}} F(x, \mu), i=1, \ldots, p,
$$

where $P_{Z_{i}}: Z \rightarrow Z_{i}$ is the projection operator onto $Z_{i}$ along $Z_{1} \oplus \cdots \oplus Z_{i-1} \oplus Z_{i+1} \oplus$ $\cdots \oplus Z_{p}$. Then, the mapping $F$ can be represented as

$$
F(x, \mu)=f_{1}(x, \mu)+\cdots+f_{p}(x, \mu)
$$

or

$$
F(x, \mu)=\left(f_{1}(x, \mu), \ldots, f_{p}(x, \mu)\right) .
$$

Denote $h=\left[h_{x}, h_{\mu}\right], h_{x} \in X, h_{\mu} \in M$.

Definition 2.1 The linear operator $\Psi_{p}=\Psi_{p}(h): X \times M \rightarrow Z$, defined by

$$
\Psi_{p}(h)=f_{1}^{\prime}\left(x^{*}, \mu^{*}\right)+f_{2}^{\prime \prime}\left(x^{*}, \mu^{*}\right)[h]+\cdots+f_{p}^{(p)}\left(x^{*}, \mu^{*}\right)[h]^{p-1}
$$

such that for any $z=(x, \mu)$

$$
\Psi_{p}(h)[z]=f_{1}^{\prime}\left(x^{*}, \mu^{*}\right)[z]+f_{2}^{\prime \prime}\left(x^{*}, \mu^{*}\right)[h][z]+\cdots+f_{p}^{(p)}\left(x^{*}, \mu^{*}\right)[h]^{p-1}[z],
$$

is called a $p$-factor operator depending of $h$ or shortly a $p$-factor operator if it is clear from the context.

Definition 2.2 We say that $F$ is completely degenerate at $\left(x^{*}, \mu^{*}\right)$ up to the order $p$ if $F^{(i)}\left(x^{*}, \mu^{*}\right)=0, i=1, \ldots, p-1$.

Remark 2.3 In the completely degenerate case, the $p$-factor operator reduces to $F^{(p)}\left(x^{*}, \mu^{*}\right)$ $[h]^{p-1}$.

Remark 2.4 For each mapping $f_{i}$, we have ([4] p. 145)

$$
f_{i}^{(k)}\left(x^{*}, \mu^{*}\right)=0, \quad k=0,1, \ldots, i-1, \quad \forall i=1, \ldots, p .
$$

Remark 2.5 For each mapping $f_{i}$, we have in the completely degenerate case

$$
f_{i}^{(i)}\left(x^{*}, \mu^{*}\right)[h]^{i-1}=P_{Z_{i}} F^{(i)}\left(x^{*}, \mu^{*}\right)[h]^{i-1}, \quad i=1, \ldots, p .
$$

This means that $f_{i}^{(i)}\left(x^{*}, \mu^{*}\right)[h]^{i-1}$ are $i$-factor operators corresponding to completely degenerate mappings $f_{i}$ up to order $i$. Therefore, the general degeneration of $F$ can be reduced to the study of completely degenerated mappings $f_{i}, i=1, \ldots, p$ and their compositions.

Let's introduce the nonlinear operator $\Psi_{p}[\cdot]^{p}$ such that

$$
\Psi_{p}[z]^{p}=f_{1}^{\prime}\left(x^{*}, \mu^{*}\right)[z]+f_{2}^{\prime \prime}\left(x^{*}, \mu^{*}\right)[z]^{2}+\cdots+f_{p}^{(p)}\left(x^{*}, \mu^{*}\right)[z]^{p}
$$

We can see that $\Psi_{p}[h]^{p}=\Psi_{p}(h)[h]$.

Definition 2.6 The p-kernel of the operator $\Psi_{p}$ is a set

$$
\begin{aligned}
& H_{p}\left(x^{*}, \mu^{*}\right)=\operatorname{Ker}^{p} \Psi_{p} \\
& \quad=\left\{h \in X \times M: f_{1}^{\prime}\left(x^{*}, \mu^{*}\right)[h]+f_{2}^{\prime \prime}\left(x^{*}, \mu^{*}\right)[h]^{2}+\cdots+f_{p}^{(p)}\left(x^{*}, \mu^{*}\right)[h]^{p}=0\right\} .
\end{aligned}
$$


Note that the following relation holds:

$$
\operatorname{Ker}^{p} \Psi_{p}=\left\{\bigcap_{i=1}^{p} \operatorname{Ker}^{i} f_{i}^{(i)}\left(x^{*}, \mu^{*}\right)\right\} .
$$

The $p$-kernel of the operator $F^{(p)}\left(x^{*}, \mu^{*}\right)$ in the completely degenerate case is a set

$$
\operatorname{Ker}^{p} F^{(p)}\left(x^{*}, \mu^{*}\right)=\left\{h \in X \times M: F^{(p)}\left(x^{*}, \mu^{*}\right)[h]^{p}=0\right\} .
$$

Definition 2.7 A mapping $F$ is called p-regularat $\left(x^{*}, \mu^{*}\right)$ along $h(p>1)$ if $\operatorname{Im} \Psi_{p}(h)=Z$ (i.e., the operator $\Psi_{p}(h)$ is surjective).

Definition 2.8 A mapping $F$ is called p-regular at $\left(x^{*}, \mu^{*}\right)(p>1)$ if it is $p$-regular along every $h \in H_{p}\left(x^{*}, \mu^{*}\right) \backslash\{0\}$ or $H_{p}\left(x^{*}, \mu^{*}\right)=\{0\}$.

Definition 2.9 Let $A \in L(X, Y)$ and $A(X)=Y$. Then define

$$
\left\|A^{-1}\right\|=\sup _{y \in Y,\|y\|=1} \inf _{x \in X}\{\|x\|: A x=y\} .
$$

Definition 2.10 Let $F: X \times M \rightarrow Z=Z_{1} \oplus \cdots \oplus Z_{p}$. The mapping $F(x, \mu)$ is called strongly $p$-regular at the point $\left(x^{*}, \mu^{*}\right)$ if there exist $\gamma>0$ and $c>0$ such that

$$
\sup _{h \in H_{\gamma}}\left\|\left\{\Psi_{p}(h)\right\}^{-1}\right\| \leq c<\infty,
$$

where

$$
\begin{aligned}
& H_{\gamma}=\left\{h=\left(h_{x}, h_{\mu}\right) \in X \times M:\left\|f_{k}^{(k)}\left(x^{*}, \mu^{*}\right)[h]^{k}\right\|_{Z_{k}} \leq \gamma,\right. \\
& \left.\forall k=1, \ldots, p, \quad\|h\|_{X \times M}=1\right\} .
\end{aligned}
$$

Define the solution set for the mapping $F$ as the set

$$
S=\left\{(x, \mu) \in X \times M: F(x, \mu)=F\left(x^{*}, \mu^{*}\right)=0\right\}
$$

and let $T_{\left(x^{*}, \mu^{*}\right)} S$ denote the tangent cone to the set $S$ at the point $\left(x^{*}, \mu^{*}\right)$, i.e.,

$$
T_{\left(x^{*}, \mu^{*}\right)} S=\left\{h \in X \times M:\left(x^{*}, \mu^{*}\right)+\varepsilon h+r(\varepsilon) \in S,\|r(\varepsilon)\|=o(\varepsilon), \varepsilon \in[0, \delta], \delta>0\right\}
$$

The following theorems describe the tangent cone to the solution set of Eq. (2.1) in the $p$-regular case.

Theorem 2.11 Let $X, M$ and $Z$ be the Banach spaces, and let the mapping $F \in C^{p}(X \times M, Z)$ be p-regular at $\left(x^{*}, \mu^{*}\right) \in X \times M$ along $h$. Then, $h \in T_{\left(x^{*}, \mu^{*}\right)} S$.

Theorem 2.12 (Generalized Lyusternik Theorem, [4]) Let X, $M$ and $Z$ be the Banach spaces, and let the mapping $F \in C^{p}(X \times M, Z)$ be p-regular at $\left(x^{*}, \mu^{*}\right) \in X \times M$. Then,

$$
T_{\left(x^{*}, \mu^{*}\right)} S=H_{p}\left(x^{*}, \mu^{*}\right) .
$$

The following Lemma (see [7]) will be used in the proof of Theorem 3.1. 
Lemma 2.13 Let $F: X \times M \rightarrow Z$, where $X, M, Z$ are Banach spaces, $z=z_{1}+\cdots+z_{p}$, $z_{i} \in Z_{i}, i=1, \ldots, p,\|h\|=1$ and

$$
\left\|\left\{\alpha_{1} f_{1}^{\prime}\left(x^{*}, \mu^{*}\right)+\alpha_{2} f_{2}^{\prime}\left(x^{*}, \mu^{*}\right)[h]+\cdots+\alpha_{p} f_{p}^{(p)}\left(x^{*}, \mu^{*}\right)[h]^{p-1}\right\}^{-1}\right\|=C<\infty .
$$

Then,

$$
\begin{aligned}
& \left\|\left\{\alpha_{1} f_{1}^{\prime}\left(x^{*}, \mu^{*}\right)+\alpha_{2} f_{2}^{\prime}\left(x^{*}, \mu^{*}\right)[t h]+\cdots+\alpha_{p} f_{p}^{(p)}\left(x^{*}, \mu^{*}\right)[t h]^{p-1}\right\}^{-1}\left(z_{1}+\cdots+z_{p}\right)\right\| \\
& \leq C\left(\frac{1}{\alpha_{1}}\left\|z_{1}\right\|+\frac{1}{\alpha_{2} t}\left\|z_{2}\right\|+\cdots+\frac{1}{\alpha_{p} t^{p-1}}\left\|z_{p}\right\|\right),
\end{aligned}
$$

where $\alpha_{i} \in \mathbb{R} \backslash\{0\}, i=1, \ldots, p, t \neq 0$.

The following Lemma will be important in the study of the surjectivity of $p$-factor operators in our Example.

Lemma 2.14 Suppose that $Y=Y_{1} \oplus Y_{2}$, where $Y_{1}$ and $Y_{2}$ are closed subspaces in $Y$, $A, B \in \mathcal{L}(X, Y)$, and $\operatorname{Im} A=Y_{1}$. Let $P_{2}$ also be the projection onto $Y_{2}$ along $Y_{1}$. Then, $\left(A+P_{2} B\right) X=Y \Leftrightarrow\left(P_{2} B\right) \operatorname{Ker} A=Y_{2}$.

This Lemma is a consequence of the following.

Lemma 2.15 Suppose that $Y=Y_{1} \oplus Y_{2}$, where $Y_{1}$ and $Y_{2}$ are closed subspaces in $Y$, $A_{1}, A_{2} \in \mathcal{L}(X, Y), A_{1} X \subset Y_{1}$, and $A_{2} X \subset Y_{2}$. Then, $\left(A_{1}+A_{2}\right) X=Y$ iff $A_{1} \operatorname{Ker} A_{2}=Y_{1}$ and $A_{2} \operatorname{Ker} A_{1}=Y_{2}$.

The proof is obvious. Lemma 2.14 follows from Lemma 2.15 if we put $A_{1}=A$ and $A_{2}=$ $P_{2} B$.

Some generalizations of the Implicit Function Theorem to the $p$-order Implicit Function Theorem for nonregular mappings and the $p$-order Implicit Function Theorem for the nontrivial kernel are in [1].

The Multivalued Contraction Mapping Theorem will be used in the proof of Theorem 3.1. Its content is available in [3].

\section{Generalization of $p$-factor Lyusternik Theorem and $p$-order Implicit Function Theorem}

In this section, we prove the Theorem, which is an analogue and generalization of the Lyusternik Theorem on the tangent cone, and discuss the existence of the continuous solution of the equation $F(x, \mu)=0$.

Let $V_{\varepsilon}\left(\mu^{*}\right)$ will be the neighborhood of $\mu^{*}$ and $C\left(V_{\varepsilon}\left(\mu^{*}\right)\right)$ will be the continuous mapping from $M$ to $X$ on neighborhood $V_{\varepsilon}\left(\mu^{*}\right)$.

Theorem 3.1 Let $F(x, \mu) \in C^{p+1}(X \times M), F: X \times M \rightarrow Z$, where $M$ is finite dimensional space, and $X$ and $Z$ are Banach spaces. Let the mappings $f_{i}(x, \mu)$ and $i=1, \ldots, p$ be defined by (2.4). Assume that $F\left(x^{*}, \mu^{*}\right)=0$ and $\forall \bar{\mu} \in M,\|\bar{\mu}\|=1$, $(0, \bar{\mu}) \in \bigcap_{k=1}^{p} \operatorname{Ker}^{k} f_{k}^{(k)}\left(x^{*}, \mu^{*}\right)$ and $F$ is strongly $p$-regular with respect to $M$ along every element $(0, \bar{\mu}), \bar{\mu} \in M$, that is,

$$
\left\|\left\{f_{1}^{\prime}\left(x^{*}, \mu^{*}\right)+f_{2}^{\prime \prime}\left(x^{*}, \mu^{*}\right)[0, \bar{\mu}]+\cdots+f_{p}^{(p)}\left(x^{*}, \mu^{*}\right)[0, \bar{\mu}]^{p-1}\right\}^{-1}\right\| \leq C .
$$


(Here, $\{\cdot\}^{-1}$ denotes the right inverse operator).

Then, there exists the continuous mapping $x=x(\mu), \mu \in V_{\varepsilon}\left(\mu^{*}\right), x(\mu) \in C\left(V_{\varepsilon}\left(\mu^{*}\right)\right)$, for sufficiently small $\varepsilon>0$, such that $F(x(\mu), \mu)=0$ and

$$
\begin{aligned}
& x(\mu)=x^{*}+\omega(\mu), \quad\|\omega(\mu)\|=o\left(\left\|\mu-\mu^{*}\right\|\right), \\
& \left\|x(\mu)-x^{*}\right\| \leq C \sum_{k=1}^{p}\left\|f_{k}\left(x^{*}, \mu\right)\right\|_{Z_{k}}^{\frac{1}{k}}, \quad \forall \mu \in V_{\varepsilon}\left(\mu^{*}\right) .
\end{aligned}
$$

Remark 3.2 The element $\mu-\mu^{*}$ plays the role of $\bar{\mu}$ in Theorem 3.1.

Proof (of Theorem 3.1) Any element $\mu \in V_{\varepsilon}\left(\mu^{*}\right)$ can be represented as $\mu^{*}+t \bar{\mu}$, where $t \in[0, \delta]$ for sufficiently small $\delta>0$. Then, we are looking for a solution of equation

$$
F\left(x^{*}+x(t \bar{\mu}), \mu^{*}+t \bar{\mu}+\tilde{\mu}(t \bar{\mu})\right)=0,
$$

where $\tilde{\mu} \in V_{\varepsilon}(0), \bar{\mu} \in M$.

Consider the multimapping $\Phi: C\left(V_{\varepsilon}(0)\right) \times V_{\varepsilon}(0) \rightarrow 2^{X \times M}$, which is defined by the formula

$$
\Phi(x, \tilde{\mu})=(x, \tilde{\mu})-\left\{\Psi_{p}(h)\right\}^{-1} F\left(x^{*}+x, \mu^{*}+t \bar{\mu}+\tilde{\mu}\right),
$$

where

$$
h=(0, t \bar{\mu}) \in \bigcap_{k=1}^{p} \operatorname{Ker}^{k} f_{k}^{(k)}\left(x^{*}, \mu^{*}\right)
$$

and the $p$-factor operator $\Psi_{p}(h): X \times M \rightarrow Z$ has the form

$$
\Psi_{p}(h)=f_{1}^{\prime}\left(x^{*}, \mu^{*}\right)+f_{2}^{\prime \prime}\left(x^{*}, \mu^{*}\right)[h]+\cdots+f_{p}^{(p)}\left(x^{*}, \mu^{*}\right)[h]^{p-1} .
$$

Note that the inverse multimapping operator is the following:

$$
\begin{aligned}
\left\{\Psi_{p}(h)\right\}^{-1}(z)= & \left\{[\xi, \eta] \in X \times M: f_{1}^{\prime}\left(x^{*}, \mu^{*}\right)[\xi, \eta]+f_{2}^{\prime \prime}\left(x^{*}, \mu^{*}\right)[h][\xi, \eta]\right. \\
& \left.+\cdots+f_{p}^{(p)}\left(x^{*}, \mu^{*}\right)[h]^{p-1}[\xi, \eta]=z\right\},
\end{aligned}
$$

where $z=z_{1}+\cdots+z_{p}$ or $z=\left(z_{1}, \ldots, z_{p}\right), z_{i} \in Z_{i}, i=1, \ldots, p$.

The "norm" of the above operator is

$$
\left\|\left\{\Psi_{p}(h)\right\}^{-1}\right\|=\sup _{\|z\|=1} \inf \left\{\|(x, \mu)\|: \Psi_{p}(h)[x, \mu]=z\right\} .
$$

We will show that there exists an element $(x, \tilde{\mu})$, such that

$$
\|(x, \tilde{\mu})\|=\|x\|+\|\tilde{\mu}\|=o(\| t \bar{\mu}) \|
$$

and $(x, \tilde{\mu}) \in \Phi(x, \tilde{\mu})$, i.e., $(x, \tilde{\mu})$ is a fixed point of the mapping $\Phi$. Then,

$$
(0,0) \in\left\{-\left\{\Psi_{p}(0, t \bar{\mu})\right\}^{-1} F\left(x^{*}+x(t \bar{\mu}), \mu^{*}+t \bar{\mu}+\tilde{\mu}(t \bar{\mu})\right)\right\} .
$$

Consequently, we will obtain

$$
F\left(x^{*}+x(t \bar{\mu}), \mu^{*}+t \bar{\mu}+\tilde{\mu}(t \bar{\mu})\right)=0
$$

and $\|(x(t \bar{\mu}), \tilde{\mu}(t \bar{\mu}))\|=o(t)$.

At the beginning, we will prove that

$$
\operatorname{dist}((0,0), \Phi(0,0))=\|\Phi(0,0)\| \leq c t^{2}=O\left(t^{2}\right)=o(t) .
$$


We have

$$
\begin{aligned}
& \Phi(0,0)=-\left\{\Psi_{p}(0, t \bar{\mu})\right\}^{-1} F\left(x^{*}, \mu^{*}+t \bar{\mu}\right) \\
& \Phi(0,0)=-\left\{\Psi_{p}(0, t \bar{\mu})\right\}^{-1}\left(f_{1}\left(x^{*}, \mu^{*}+t \bar{\mu}\right)+f_{2}\left(x^{*}, \mu^{*}+t \bar{\mu}\right)+\cdots+f_{p}\left(x^{*}, \mu^{*}+t \bar{\mu}\right)\right)
\end{aligned}
$$

and

$$
\begin{aligned}
\|\Phi(0,0)\|= & \|-\left\{\Psi_{p}(0, t \bar{\mu})\right\}^{-1}\left(f_{1}\left(x^{*}, \mu^{*}+t \bar{\mu}\right)\right. \\
& \left.+f_{2}\left(x^{*}, \mu^{*}+t \bar{\mu}\right)+\cdots+f_{p}\left(x^{*}, \mu^{*}+t \bar{\mu}\right)\right) \| .
\end{aligned}
$$

By Lemma 2.13, we obtain

$$
\|\Phi(0,0)\| \leq \| c\left(f_{1}\left(x^{*}, \mu^{*}+t \bar{\mu}\right)\left\|+\frac{c}{t}\right\| f_{2}\left(x^{*}, \mu^{*}+t \bar{\mu}\right)\left\|+\cdots+\frac{c}{t^{p-1}}\right\| f_{p}\left(x^{*}, \mu^{*}+t \bar{\mu}\right) \| .\right.
$$

We apply the Taylor formula to the expressions $f_{i}\left(x^{*}, \mu^{*}+t \bar{\mu}\right)$ for $i=1, \ldots p$, and we have

$$
\begin{aligned}
& \|\Phi(0,0)\| \leq c\left\|f_{1}\left(x^{*}, \mu^{*}\right)+f_{1}^{\prime}\left(x^{*}, \mu^{*}\right)[t \cdot 0, t \bar{\mu}]+O_{Z}\left(t^{2}\right)\right\| \\
& +\frac{c}{t}\left\|f_{2}\left(x^{*}, \mu^{*}\right)+f_{2}^{\prime}\left(x^{*}, \mu^{*}\right)[t \cdot 0, t \bar{\mu}]+\frac{1}{2 !} f_{2}^{\prime \prime}\left(x^{*}, \mu^{*}\right)[t \cdot 0, t \bar{\mu}]^{2}+O_{Z}\left(t^{3}\right)\right\| \\
& +\cdots+\frac{c}{t^{p-1}} \| f_{p}\left(x^{*}, \mu^{*}\right)+f_{p}^{\prime}\left(x^{*}, \mu^{*}\right)[t \cdot 0, t \bar{\mu}]+\cdots+\frac{1}{p !} f_{p}^{(p)}\left(x^{*}, \mu^{*}\right)[t \cdot 0, t \bar{\mu}]^{p} \\
& +O_{Z}\left(t^{p+1}\right) \| .
\end{aligned}
$$

Therefore, based on the relation (2.9), we estimate

$$
\|\Phi(0,0)\| \leq c t^{2}+\frac{c}{t} t^{3}+\cdots+\frac{c}{t^{p-1}} t^{p+1}=p c t^{2}
$$

and

$$
\|\Phi(0,0)\|=O\left(t^{2}\right)=o(t) .
$$

Now, we show that for any $\left(x_{1}, \mu_{1}\right),\left(x_{2}, \mu_{2}\right) \in V_{O\left(t^{2}\right)}(0,0)$, the following estimation holds:

$$
\operatorname{dist}_{\mathrm{H}}\left(\Phi\left(x_{1}, \mu_{1}\right), \Phi\left(x_{2}, \mu_{2}\right)\right) \leq \theta\left\|\left(x_{1}, \mu_{1}\right)-\left(x_{2}, \mu_{2}\right)\right\|,
$$

where $0<\theta<1$.

Note, at the beginning, that

$$
\Psi_{p}(t h) \Phi\left(x_{1}, \mu_{1}\right)=\Psi_{p}(t h)\left(x_{1}, \mu_{1}\right)-F\left(\left(x^{*}, \mu^{*}\right)+t h+\left(x_{1}, \mu_{1}\right)\right)
$$

and

$$
\Psi_{p}(t h) \Phi\left(x_{2}, \mu_{2}\right)=\Psi_{p}(t h)\left(x_{2}, \mu_{2}\right)-F\left(\left(x^{*}, \mu^{*}\right)+t h+\left(x_{2}, \mu_{2}\right)\right) .
$$

Let $\left(z_{1}, \xi_{1}\right) \in \Phi\left(x_{1}, \mu_{1}\right),\left(z_{2}, \xi_{2}\right) \in \Phi\left(x_{2}, \mu_{2}\right)$. Then, we have

$$
\begin{aligned}
\operatorname{dist}_{\mathrm{H}} & \left(\Phi\left(x_{1}, \mu_{1}\right), \Phi\left(x_{2}, \mu_{2}\right)\right) \\
= & \inf \left\{\left\|\left(z_{1}, \xi_{1}\right)-\left(z_{2}, \xi_{2}\right)\right\|:\left(z_{i}, \xi_{i}\right) \in \Phi\left(x_{i}, \mu_{i}\right), i=1,2\right\} \\
= & \inf \left\{\left\|\left(z_{1}, \xi_{1}\right)-\left(z_{2}, \xi_{2}\right)\right\|: \Psi_{p}(t h)\left(\left(z_{1}, \xi_{1}\right)-\left(z_{2}, \xi_{2}\right)\right)\right. \\
= & \Psi_{p}(t h)\left(\left(x_{1}, \mu_{1}\right)-\left(x_{2}, \mu_{2}\right)\right) \\
& \left.-\left[F\left(\left(x^{*}, \mu^{*}\right)+t h+\left(x_{1}, \mu_{1}\right)\right)-F\left(\left(x^{*}, \mu^{*}\right)+t h+\left(x_{2}, \mu_{2}\right)\right)\right]\right\}
\end{aligned}
$$




$$
\begin{aligned}
= & \inf \left\{\|(z, \xi)\|: \Psi_{p}(t h)(z, \xi)\right. \\
= & \Psi_{p}(t h)\left(\left(x_{1}, \mu_{1}\right)-\left(x_{2}, \mu_{2}\right)\right) \\
& \left.-\left[F\left(\left(x^{*}, \mu^{*}\right)+t h+\left(x_{1}, \mu_{1}\right)\right)-F\left(\left(x^{*}, \mu^{*}\right)+t h+\left(x_{2}, \mu_{2}\right)\right)\right]\right\} \\
= & \inf \left\{\|(z, \xi)\|: \Psi_{p}(t h)(z, \xi)=\Psi_{p}(t h)\left(\left(x_{1}, \mu_{1}\right)-\left(x_{2}, \mu_{2}\right)\right)\right. \\
& \left.-\left[\sum_{i=1}^{p}\left(f_{i}\left(\left(x^{*}, \mu^{*}\right)+t h+\left(x_{1}, \mu_{1}\right)\right)-f_{i}\left(\left(x^{*}, \mu^{*}\right)+t h+\left(x_{2}, \mu_{2}\right)\right)\right)\right]\right\} \\
= & \inf \left\{\|\left\{\Psi_{p}(t h)\right\}^{-1}\left[f_{1}^{\prime}\left(x^{*}, \mu^{*}\right)\left(\left(x_{1}, \mu_{1}\right)-\left(x_{2}, \mu_{2}\right)\right)\right.\right. \\
& +\sum_{i=2}^{p} f_{i}^{(i)}\left(x^{*}, \mu^{*}\right)[t h]^{i-1}\left(\left(x_{1}, \mu_{1}\right)-\left(x_{2}, \mu_{2}\right)\right) \\
& \left.\left.-\left[\sum_{i=1}^{p}\left(f_{i}\left(\left(x^{*}, \mu^{*}\right)+t h+\left(x_{1}, \mu_{1}\right)\right)-f_{i}\left(\left(x^{*}, \mu^{*}\right)+t h+\left(x_{2}, \mu_{2}\right)\right)\right)\right]\right] \|\right\} \\
= & \inf \left\{\|\left\{\Psi_{p}(t h)\right\}^{-1}\right. \\
& {\left[\left[f_{1}^{\prime}\left(x^{*}, \mu^{*}\right)\left(\left(x_{1}, \mu_{1}\right)-\left(x_{2}, \mu_{2}\right)\right)-\left(f_{1}\left(\left(x^{*}, \mu^{*}\right)+t h+\left(x_{1}, \mu_{1}\right)\right)\right.\right.\right.} \\
& \left.-f_{1}\left(\left(x^{*}, \mu^{*}\right)+t h+\left(x_{2}, \mu_{2}\right)\right)\right] \\
& +\sum_{i=2}^{p}\left[f_{i}^{(i)}\left(x^{*}, \mu^{*}\right)[t h]^{i-1}\left(\left(x_{1}, \mu_{1}\right)-x_{2}, \mu_{2}\right)\right)-\left(f_{i}\left(\left(x^{*}, \mu^{*}\right)+t h+\left(x_{1}, \mu_{1}\right)\right)\right. \\
& \left.\left.-\left(f_{i}\left(\left(x^{*}, \mu^{*}\right)+t h+\left(x_{2}, \mu_{2}\right)\right)\right]\right] \|\right\} .
\end{aligned}
$$

By Lemma 2.13, we obtain the following estimation:

$$
\begin{aligned}
\operatorname{dist}_{\mathrm{H}} & \left(\Phi\left(x_{1}, \mu_{1}\right), \Phi\left(x_{2}, \mu_{2}\right)\right) \\
\leq & c \| f_{1}\left(\left(x^{*}, \mu^{*}\right)+t h+\left(x_{1}, \mu_{1}\right)\right)-f_{1}\left(\left(x^{*}, \mu^{*}\right)+t h+\left(x_{2}, \mu_{2}\right)\right) \\
& -f_{1}^{\prime}\left(x^{*}, \mu^{*}\right)\left(\left(x_{1}, \mu_{1}\right)-\left(x_{2}, \mu_{2}\right)\right) \| \\
& +\frac{c}{t} \| f_{2}\left(\left(x^{*}, \mu^{*}\right)+t h+\left(x_{1}, \mu_{1}\right)\right)-f_{2}\left(\left(x^{*}, \mu^{*}\right)+t h+\left(x_{2}, \mu_{2}\right)\right) \\
& -f_{2}^{\prime \prime}[t h]\left(x^{*}, \mu^{*}\right)\left(\left(x_{1}, \mu_{1}\right)-\left(x_{2}, \mu_{2}\right)\right) \| \\
& +\cdots+\frac{c}{t^{p-1}} \| f_{p}\left(\left(x^{*}, \mu^{*}\right)+t h+\left(x_{1}, \mu_{1}\right)\right)-f_{p}\left(\left(x^{*}, \mu^{*}\right)+t h+\left(x_{2}, \mu_{2}\right)\right) \\
& -f_{p}^{(p)}[t h]^{p-1}\left(x^{*}, \mu^{*}\right)\left(\left(x_{1}, \mu_{1}\right)-\left(x_{2}, \mu_{2}\right)\right) \| \\
= & A_{1}+A_{2}+\cdots+A_{p},
\end{aligned}
$$

where

$$
\begin{aligned}
A_{1}= & c \| f_{1}\left(\left(x^{*}, \mu^{*}\right)+t h+\left(x_{1}, \mu_{1}\right)\right)-f_{1}\left(\left(x^{*}, \mu^{*}\right)+t h+\left(x_{2}, \mu_{2}\right)\right) \\
& -f_{1}^{\prime}\left(x^{*}, \mu^{*}\right)\left(\left(x_{1}, \mu_{1}\right)-\left(x_{2}, \mu_{2}\right)\right) \|, \\
A_{2}= & \frac{c}{t} \| f_{2}\left(\left(x^{*}, \mu^{*}\right)+t h+\left(x_{1}, \mu_{1}\right)\right)-f_{2}\left(\left(x^{*}, \mu^{*}\right)+t h+\left(x_{2}, \mu_{2}\right)\right) \\
& -f_{2}^{\prime \prime}[t h]\left(x^{*}, \mu^{*}\right)\left(\left(x_{1}, \mu_{1}\right)-\left(x_{2}, \mu_{2}\right)\right) \|, \\
& \vdots \\
A_{p}= & \frac{c}{t^{p-1}} \| f_{p}\left(\left(x^{*}, \mu^{*}\right)+t h+\left(x_{1}, \mu_{1}\right)\right)-f_{p}\left(\left(x^{*}, \mu^{*}\right)+t h+\left(x_{2}, \mu_{2}\right)\right) \\
& -f_{p}^{(p)}[t h]^{p-1}\left(x^{*}, \mu^{*}\right)\left(\left(x_{1}, \mu_{1}\right)-\left(x_{2}, \mu_{2}\right)\right) \| .
\end{aligned}
$$


To the component $A_{1}$, we apply the Mean Value Theorem and then the Taylor formula to the expression $f_{1}^{\prime}\left[\left(x^{*}, \mu^{*}\right)+t h+\left(x_{2}, \mu_{2}\right)+\bar{\theta}\left(\left(x_{1}, \mu_{1}\right)-\left(x_{2}, \mu_{2}\right)\right)\right]$. We have

$$
\begin{aligned}
A_{1} \leq & c \sup _{\bar{\theta} \in[0,1]}\left\|f_{1}^{\prime}\left(\left(x^{*}, \mu^{*}\right)+t h+\left(x_{2}, \mu_{2}\right)+\bar{\theta}\left(\left(x_{1}, \mu_{1}\right)-\left(x_{2}, \mu_{2}\right)\right)\right)-f_{1}^{\prime}\left(x^{*}, \mu^{*}\right)\right\| \\
& \cdot\left\|\left(x_{1}, \mu_{1}\right)-\left(x_{2}, \mu_{2}\right)\right\| \\
= & c \sup _{\bar{\theta} \in[0,1]}\left\|f_{1}^{\prime}\left(x^{*}, \mu^{*}\right)+O_{Z}(t)-f_{1}^{\prime}\left(x^{*}, \mu^{*}\right)\right\|\left\|\left(x_{1}, \mu_{1}\right)-\left(x_{2}, \mu_{2}\right)\right\| \\
= & c \sup _{\bar{\theta} \in[0,1]}\left\|O_{Z}(t)\right\|\left\|\left(x_{1}, \mu_{1}\right)-\left(x_{2}, \mu_{2}\right)\right\| \leq c c_{1} t\left\|\left(x_{1}, \mu_{1}\right)-\left(x_{2}, \mu_{2}\right)\right\| \\
= & k_{1} t\left\|\left(x_{1}, \mu_{1}\right)-\left(x_{2}, \mu_{2}\right)\right\|,
\end{aligned}
$$

where $k_{1}=c c_{1}$. Now, put $\theta_{1}=k_{1} t$, where $t \in(0, \delta), \delta$ is sufficiently small, and then,

$$
A_{1} \leq \theta_{1}\left\|\left(x_{1}, \mu_{1}\right)-\left(x_{2}, \mu_{2}\right)\right\| .
$$

To the component $A_{2}$, we apply the Mean Value Theorem and then the Taylor formula to the expression $f_{2}^{\prime}\left[\left(x^{*}, \mu^{*}\right)+t h+\left(x_{2}, \mu_{2}\right)+\bar{\theta}\left(\left(x_{1}, \mu_{1}\right)-\left(x_{2}, \mu_{2}\right)\right)\right]$. We now have

$$
\begin{aligned}
A_{2} \leq & \frac{c}{t} \sup _{\bar{\theta} \in[0,1]}\left\|f_{2}^{\prime}\left[\left(x^{*}, \mu^{*}\right)+t h+\left(x_{2}, \mu_{2}\right)+\bar{\theta}\left(\left(x_{1}, \mu_{1}\right)-\left(x_{2}, \mu_{2}\right)\right)\right]-f_{2}^{\prime \prime}\left(x^{*}, \mu^{*}\right)[t h]\right\| \\
& \cdot\left\|\left(x_{1}, \mu_{1}\right)-\left(x_{2}, \mu_{2}\right)\right\| \\
= & \frac{c}{t} \sup _{\bar{\theta} \in[0,1]} \| f_{2}^{\prime}\left(x^{*}, \mu^{*}\right)+f_{2}^{\prime \prime}\left(x^{*}, \mu^{*}\right)\left[t h+\left(x_{2}, \mu_{2}\right)+\bar{\theta}\left(\left(x_{1}, \mu_{1}\right)-\left(x_{2}, \mu_{2}\right)\right)\right] \\
& +O_{Z}\left(t^{2}\right)-f_{2}^{\prime \prime}\left(x^{*}\right)[t h]\|\|\left(x_{1}, \mu_{1}\right)-\left(x_{2}, \mu_{2}\right) \| \\
= & \frac{c}{t} \sup _{\bar{\theta} \in[0,1]} \| f_{2}^{\prime}\left(x^{*}, \mu^{*}\right)+f_{2}^{\prime \prime}\left(x^{*}, \mu^{*}\right)[t h] \\
& +f_{2}^{\prime \prime}\left(x^{*}, \mu^{*}\right)\left[\left(x_{2}, \mu_{2}\right)+\bar{\theta}\left(\left(x_{1}, \mu_{1}\right)-\left(x_{2}, \mu_{2}\right)\right)\right] \\
& +O_{Z}\left(t^{2}\right)-f_{2}^{\prime \prime}\left(x^{*}, \mu_{*}\right)[t h]\|\|\left(x_{1}, \mu_{1}\right)-\left(x_{2}, \mu_{2}\right) \| .
\end{aligned}
$$

Since $f_{2}^{\prime}\left(x^{*}, \mu^{*}\right)=0$ [see (2.9)], we obtain

$$
\begin{aligned}
A_{2} \leq & \frac{c}{t} \sup _{\bar{\theta} \in[0,1]}\left\|f_{2}^{\prime \prime}\left(x^{*}, \mu^{*}\right)\left[\left(x_{2}, \mu_{2}\right)+\bar{\theta}\left(\left(x_{1}, \mu_{1}\right)-\left(x_{2}, \mu_{2}\right)\right)\right]+O_{Z}\left(t^{2}\right)\right\| \\
& \left.\cdot \|\left(x_{1}, \mu_{1}\right)-\left(x_{2}, \mu_{2}\right)\right) \|,
\end{aligned}
$$

and therefore,

$$
\begin{aligned}
A_{2} \leq & \frac{c}{t} \sup _{\bar{\theta} \in[0,1]}\left(\left\|f_{2}^{\prime \prime}\left(x^{*}, \mu^{*}\right)\left[\left(x_{2}, \mu_{2}\right)+\bar{\theta}\left(\left(x_{1}, \mu_{1}\right)-\left(x_{2}, \mu_{2}\right)\right)\right]\right\|+\left\|O_{Z}\left(t^{2}\right)\right\|\right) \\
& \left.\cdot \|\left(x_{1}, \mu_{1}\right)-\left(x_{2}, \mu_{2}\right)\right) \|,
\end{aligned}
$$

Finally, using the fact that the rank of expression $f_{2}^{\prime \prime}\left(x^{*}, \mu^{*}\right)\left[\left(x_{2}, \mu_{2}\right)+\bar{\theta}\left(\left(x_{1}, \mu_{1}\right)-\right.\right.$ $\left.\left.\left(x_{2}, \mu_{2}\right)\right)\right]$ is $t^{2}$, the rank of $O_{Z}\left(t^{2}\right)$ is $t^{2}$ and the properties of the norm, we conclude that

$$
A_{2} \leq \frac{c}{t}\left(d_{1} t^{2}+d_{2} t^{2}\right)\left\|\left(x_{1}, \mu_{1}\right)-\left(x_{2}, \mu_{2}\right)\right\| \leq 2 k_{2} t\left\|\left(x_{1}, \mu_{1}\right)-\left(x_{2}, \mu_{2}\right)\right\|,
$$

where $k_{2}=\max \left\{c d_{1}, c d_{2}\right\}$. We can substitute $\theta_{2}=2 k_{2} t$, and then,

$$
\left.A_{2} \leq \theta_{2} \|\left(x_{1}, \mu_{1}\right)-\left(x_{2}, \mu_{2}\right)\right) \|,
$$

where $t \in(0, \delta)$, and $\delta>0$ is sufficiently small. 
Similarly, we will now evaluate the component $A_{p}$ also using the Mean Value Theorem and the extension of expression $f_{p}^{\prime}\left[\left(x^{*}, \mu^{*}\right)+t h+\left(x_{2}, \mu_{2}\right)+\bar{\theta}\left(\left(x_{1}, \mu_{1}\right)-\left(x_{2}, \mu_{2}\right)\right)\right]$ in the Taylor formula.

Therefore, let us note that

$$
\begin{aligned}
A_{p} \leq & \frac{c}{t^{p-1}} \sup _{\bar{\theta} \in[0,1]} \| f_{p}^{\prime}\left[\left(x^{*}, \mu^{*}\right)+t h+\left(x_{2}, \mu_{2}\right)+\bar{\theta}\left(\left(x_{1}, \mu_{1}\right)-\left(x_{2}, \mu_{2}\right)\right)\right] \\
& \left.-\frac{1}{(p-1) !} f_{p}^{(p)}\left(x^{*}, \mu^{*}\right)[t h]^{p-1}\|\|\left(x_{1}, \mu_{1}\right)-\left(x_{2}, \mu_{2}\right)\right) \|
\end{aligned}
$$

By the Taylor formula, we have

$$
\begin{aligned}
& f_{p}^{\prime}\left[\left(x^{*}, \mu^{*}\right)+t h+\left(x_{2}, \mu_{2}\right)+\bar{\theta}\left(\left(x_{1}, \mu_{1}\right)-\left(x_{2}, \mu_{2}\right)\right)\right] \\
& \quad=f_{p}^{\prime}\left(x^{*}, \mu^{*}\right)+f_{p}^{\prime \prime}\left(x^{*}, \mu^{*}\right)\left[t h+\left(x_{2}, \mu_{2}\right)+\bar{\theta}\left(\left(x_{1}, \mu_{1}\right)-\left(x_{2}, \mu_{2}\right)\right)\right] \\
& +\cdots+\frac{1}{(p-1) !} f_{p}^{(p)}\left(x^{*}, \mu^{*}\right)\left[t h+\left(x_{2}, \mu_{2}\right)+\bar{\theta}\left(\left(x_{1}, \mu_{1}\right)-\left(x_{2}, \mu_{2}\right)\right)\right]^{p-1}+O_{Z}\left(t^{p}\right) .
\end{aligned}
$$

According to (2.9), the mapping $f_{p}^{(i)}\left(x^{*}, \mu^{*}\right)=0$, for $i=1,2, \ldots, p-1$. Then, we obtain

$$
\begin{aligned}
A_{p} \leq & \frac{c}{t^{p-1}} \sup _{\bar{\theta} \in[0,1]} \| \frac{1}{(p-1) !} f_{p}^{(p)}\left(x^{*}, \mu^{*}\right)\left[t h+\left(x_{2}, \mu_{2}\right)+\bar{\theta}\left(\left(x_{1}, \mu_{1}\right)-\left(x_{2}, \mu_{2}\right)\right)\right]^{p-1} \\
& \left.+O_{Z}\left(t^{p}\right)-\frac{1}{(p-1) !} f_{p}^{(p)}\left(x^{*}, \mu^{*}\right)[t h]^{p-1}\|\|\left(x_{1}, \mu_{1}\right)-\left(x_{2}, \mu_{2}\right)\right) \| \\
= & \frac{c}{t^{p-1}} \sup _{\bar{\theta} \in[0,1]} \| \frac{1}{(p-1) !} f_{p}^{(p)}\left(x^{*}, \mu^{*}\right)[t h]^{p-1} \\
& +\frac{1}{(p-1) !} f_{p}^{(p)}\left(x^{*}, \mu^{*}\right)[t h]^{p-2}\left[\left(x_{2}, \mu_{2}\right)+\bar{\theta}\left(\left(x_{1}, \mu_{1}\right)-\left(x_{2}, \mu_{2}\right)\right)\right] \\
& \left.+O_{Z}\left(t^{p}\right)-\frac{1}{(p-1) !} f_{p}^{(p)}\left(x^{*}, \mu^{*}\right)[t h]^{p-1}\|\|\left(x_{1}, \mu_{1}\right)-\left(x_{2}, \mu_{2}\right)\right) \|,
\end{aligned}
$$

i.e.,

$$
\begin{aligned}
A_{p} \leq & \frac{c}{t^{p-1}} \sup _{\bar{\theta} \in[0,1]} \| \frac{1}{(p-1) !} f_{p}^{(p)}\left(x^{*}, \mu^{*}\right)[t h]^{p-2}\left[\left(x_{2}, \mu_{2}\right)+\bar{\theta}\left(\left(x_{1}, \mu_{1}\right)-\left(x_{2}, \mu_{2}\right)\right)\right] \\
& \left.+O_{Z}\left(t^{p}\right)\|\|\left(x_{1}, \mu_{1}\right)-\left(x_{2}, \mu_{2}\right)\right) \| .
\end{aligned}
$$

Then, according to the property of a norm, we obtain

$$
\begin{aligned}
A_{p} \leq & \frac{c}{t^{p-1}} \sup _{\bar{\theta} \in[0,1]}\left(\left\|\frac{1}{(p-1) !} f_{p}^{(p)}\left(x^{*}, \mu^{*}\right)[t h]^{p-2}\left[\left(x_{2}, \mu_{2}\right)+\bar{\theta}\left(\left(x_{1}, \mu_{1}\right)-\left(x_{2}, \mu_{2}\right)\right)\right]\right\|\right. \\
& \left.+\left\|O_{Z}\left(t^{p}\right)\right\|\right)\left\|\left(x_{1}, \mu_{1}\right)-\left(x_{2}, \mu_{2}\right)\right\| .
\end{aligned}
$$

Since $\left(x_{1}, \mu_{1}\right),\left(x_{2}, \mu_{2}\right) \in V_{O\left(t^{2}\right)}(0,0)$, then

$$
\begin{aligned}
A_{p} & \leq \frac{c}{t^{p-1}}\left(\bar{d}_{1} t^{p-2+2}+\bar{d}_{2} t^{p}\right)\left\|\left(x_{1}, \mu_{1}\right)-\left(x_{2}, \mu_{2}\right)\right\| \\
& \leq 2 k_{p} t\left\|\left(x_{1}, \mu_{1}\right)-\left(x_{2}, \mu_{2}\right)\right\|=\theta_{p}\left\|\left(x_{1}, \mu_{1}\right)-\left(x_{2}, \mu_{2}\right)\right\|,
\end{aligned}
$$

where $k_{2}=\max \left\{c \bar{d}_{1}, c \bar{d}_{2}\right\}, t \in(0, \delta), \delta>0$ is sufficiently small and $\theta_{p}=2 k_{p} t$. 
Substituting $\theta=\theta_{1}+\theta_{2}+\cdots+\theta_{p}=\bar{c} t, \bar{c}>0$, we obtain

$$
\operatorname{dist}_{\mathrm{H}}\left(\Phi\left(x_{1}, \mu_{1}\right), \Phi\left(x_{2}, \mu_{2}\right)\right) \leq \theta\left\|\left(x_{1}, \mu_{1}\right)-\left(x_{2}, \mu_{2}\right)\right\|,
$$

and $0<\theta<1$.

According to the Multivalued Contraction Principle, we will show that

$$
\varrho((0,0), \Phi(0,0))=\|\Phi(0,0)\|<(1-\theta) \varepsilon
$$

where $\theta=\overline{\bar{c}} t, \varepsilon=4 p c t^{2}$, for $t$ is sufficiently small.

We can put $0<\theta=\overline{\bar{c}} t<\frac{1}{2}$. This inequality is equivalent to $1<2(1-\overline{\bar{c}} t)$. From this and inequality $\|\Phi(0,0)\| \leq p c t^{2}$, we obtain

$$
\|\Phi(0,0)\| \leq p c t^{2} \leq 2 p(1-\overline{\bar{c}} t) c t^{2}<(1-\overline{\bar{c}} t) 4 p c t^{2}=(1-\theta) \varepsilon,
$$

that which was to be proven.

Therefore, we proved that the mapping $\Phi$ is a contraction in the set $V\left((0,0), c t^{2}\right)$. By the Multivalued Contraction Principle for $\left(z_{0}, \mu_{0}\right)=(0,0)$, it follows that there exists element $(x, \tilde{\mu})$, such that

$$
\|(x, \tilde{\mu})\| \leq \frac{2}{1-\theta}\|\Phi(0,0)\| \leq c t^{2},
$$

i.e., $\|(x, \tilde{\mu})\|=o(t)$ and $(x, \tilde{\mu}) \in \Phi(x, \tilde{\mu})$. Therefore, $(x, \tilde{\mu})$ is a fixed point of the mapping $\Phi$. Then,

$$
(0,0) \in\left\{-\left\{\Psi_{p}(0, t \bar{\mu})\right\}^{-1} F\left(x^{*}+x(t \bar{\mu}), \mu^{*}+t \bar{\mu}+\tilde{\mu}(t \bar{\mu})\right)\right\} .
$$

Consequently, we obtain

$$
F\left(x^{*}+x(t \bar{\mu}), \mu^{*}+t \bar{\mu}+\tilde{\mu}(t \bar{\mu})\right)=0
$$

and $\|(x(t \bar{\mu}), \tilde{\mu}(t \bar{\mu}))\|=o(t)$.

Summarizing, we showed that for parameter $\mu^{*}+t \bar{\mu}$, we have the solution $\left(x^{*}+\right.$ $\left.x(t \bar{\mu}), \mu^{*}+t \bar{\mu}+\tilde{\mu}(t \bar{\mu})\right)$ of equation $F(x, \mu)=0$, i.e.,

$$
F\left(x^{*}+x(t \bar{\mu}), \mu^{*}+t \bar{\mu}+\tilde{\mu}(t \bar{\mu})\right)=0 .
$$

Now, without loss of generality, let the set $M$ be equal to $\mathbb{R}^{2}$, and let us take any $\mu$ that is a sufficiently small element from $\mathbb{R}^{2}$. For such $\mu$, there exists $\overline{\bar{\mu}}(\mu)$ such that

$$
\|\overline{\bar{\mu}}(\mu)-\mu\|=o(\mu)
$$

and as we showed earlier,

$$
F\left(x^{*}+x(\overline{\bar{\mu}}(\mu)), \mu^{*}+\overline{\bar{\mu}}(\mu)+\tilde{\mu}(\overline{\bar{\mu}}(\mu))\right)=0 .
$$

Now is the important moment. We return to $\mu$. From the assumptions, we put $\mu=\mu^{*}+$ $\overline{\bar{\mu}}(\mu)+\tilde{\mu}(\overline{\bar{\mu}}(\mu))$, and marking $x^{*}+x(\overline{\bar{\mu}}(\mu))$ by $x(\mu)$, we obtain the equation

$$
F(x(\mu), \mu)=0 .
$$

The above equation holds since we took any $\bar{\mu}$ from $\mathbb{R}^{2}$, and we proved that (3.14) holds for any $\bar{\mu}$. Therefore, based on the above, for any $\mu$ from $\mathbb{R}^{2}$, there exists $\overline{\bar{\mu}}(\mu)$ such that the contraction process is beginning in the point $\left(0, \mu^{*}+\overline{\bar{\mu}}(\mu)\right)$ and generates solutions (3.16) and finally (3.17).

This ends the first part of the proof (we proved the existence of solutions). 
Let $U$ be a sufficiently small neighbourhood of $\left(x^{*}, \mu^{*}\right)$.

Let us take $\mu^{*}+t \bar{\mu}$, where $t>0$ is sufficiently small and put

$$
h=\frac{(0, t \bar{\mu})}{\|t \bar{\mu}\|}=(0, \bar{\mu}) \in \bigcap_{k=1}^{p} \operatorname{Ker}^{k} f_{k}^{(k)}\left(x^{*}, \mu^{*}\right),
$$

where $\|\bar{\mu}\|=1$. Then, for any $k \leq p$

$$
f_{k}^{(k)}\left(x^{*}, \mu^{*}\right)[0, \bar{\mu}]^{k}=0, \quad k=1, \ldots, p .
$$

We showed in Eq. (3.5) that

$$
\|\Phi(0,0)\| \leq \| c\left(f_{1}\left(x^{*}, \mu^{*}+t \bar{\mu}\right)\left\|+\frac{c}{t}\right\| f_{2}\left(x^{*}, \mu^{*}+t \bar{\mu}\right)\left\|+\cdots+\frac{c}{t^{p-1}}\right\| f_{p}\left(x^{*}, \mu^{*}+t \bar{\mu}\right) \|\right.
$$

or

$$
\|\Phi(0,0)\| \leq c \sum_{k=1}^{p}\left\|\frac{f_{k}\left(x^{*}, \mu^{*}+t \bar{\mu}\right)}{t^{k-1}}\right\| .
$$

We have for $\mu^{*}+t \bar{\mu}$

$$
\left\|x\left(\mu^{*}+t \bar{\mu}\right)-x^{*}\right\| \leq\left\|\left(x\left(\mu^{*}+t \bar{\mu}\right)-x^{*}, t \bar{\mu}\right)\right\| \leq C\|\Phi(0,0)\| .
$$

Let us note that the following inequality holds:

$$
\left\|f_{k}\left(x^{*}, \mu^{*}+t \bar{\mu}\right)-f_{k}\left(x^{*}, \mu^{*}\right)\right\| \leq C_{k}\left\|f_{k}\left(x^{*}, \mu^{*}+t \bar{\mu}\right)-f_{k}\left(x^{*}, \mu^{*}\right)\right\|^{\frac{1}{k}}\|(0, t \bar{\mu})\|^{k-1}
$$

or

$$
\left\|f_{k}\left(x^{*}, \mu^{*}+t \bar{\mu}\right)-f_{k}\left(x^{*}, \mu^{*}\right)\right\|^{k-1} \leq C_{k}^{k}\|(0, t \bar{\mu})\|^{k(k-1)} .
$$

This is true because by the Taylor expansion and relations (2.9), we have

$$
\begin{aligned}
& \| f_{k}\left(x^{*}, \mu^{*}\right)+f_{k}^{\prime}\left(x^{*}, \mu^{*}\right)[0, t \bar{\mu}] \\
& \quad+\cdots+\frac{1}{(k-1) !} f_{k}^{(k)}\left(x^{*}, \mu^{*}\right)[0, t \bar{\mu}]^{k}+O\left(t^{k+1}\right)-f_{k}\left(x^{*}, \mu^{*}\right) \|^{k-1} \\
& =\left\|\frac{1}{(k-1) !} f_{k}^{(k)}\left(x^{*}, \mu^{*}\right)[0, t \bar{\mu}]^{k}+O\left(t^{k+1}\right)\right\|^{k-1} \leq C t^{k(k-1)}=C\|(0, t \bar{\mu})\|^{k(k-1)} .
\end{aligned}
$$

Consequently, the relation (3.21) is satisfied. Therefore, we have

$$
\begin{aligned}
\left\|x\left(\mu^{*}+t \bar{\mu}\right)-x^{*}\right\| & \leq C\|\Phi(0,0)\| \leq C c \sum_{k=1}^{p}\left\|\frac{f_{k}\left(x^{*}, \mu^{*}+t \bar{\mu}\right)}{t^{k-1}}\right\| \\
& \leq \bar{C} \sum_{k=1}^{p} \frac{\left\|f_{k}\left(x^{*}, \mu^{*}+t \bar{\mu}\right)-f_{k}\left(x^{*}, \mu^{*}\right)\right\|^{\frac{1}{k}}}{\left\|t^{k-1}\right\|}\|(0, t \bar{\mu})\|^{k-1} .
\end{aligned}
$$

and

$$
\left\|x\left(\mu^{*}+t \bar{\mu}\right)-x^{*}\right\| \leq \bar{C} \sum_{k=1}^{p}\left\|f_{k}\left(x^{*}, \mu^{*}+t \bar{\mu}\right)\right\|^{\frac{1}{k}}
$$


since $f_{k}\left(x^{*}, \mu^{*}\right)=0$.

Next, from (3.23), for any sufficiently small $\mu$, there exists $\overline{\bar{\mu}}(\mu)$, such that

$$
\left\|x\left(\mu^{*}+\overline{\bar{\mu}}(\mu)\right)-x^{*}\right\| \leq \bar{C} \sum_{k=1}^{p}\left\|f_{k}\left(x^{*}, \mu^{*}+\overline{\bar{\mu}}(\mu)\right)\right\|^{\frac{1}{k}} .
$$

From this, we have

$$
\begin{aligned}
\left\|x(\mu)-x^{*}\right\| \leq \overline{\bar{C}} \sum_{k=1}^{p}\left\|f_{k}\left(x^{*}, \mu^{*}+\overline{\bar{\mu}}(\mu)\right)\right\|^{\frac{1}{k}} & \leq \overline{\bar{C}} \sum_{k=1}^{p}\left\|f_{k}\left(x^{*}, \mu^{*}+\overline{\bar{\mu}}(\mu)+\tilde{\mu}(\mu)\right)\right\|^{\frac{1}{k}} \\
& =\overline{\bar{C}} \sum_{k=1}^{p}\left\|f_{k}\left(x^{*}, \mu\right)\right\|^{\frac{1}{k}},
\end{aligned}
$$

where

$$
\mu=\mu^{*}+\overline{\bar{\mu}}(\mu)+\tilde{\mu}(\mu) .
$$

Here, we used the following fact:

$$
\left\|f_{k}\left(x^{*}, \mu^{*}+\overline{\bar{\mu}}(\mu)\right)\right\| \leq 2\left\|f_{k}\left(x^{*}, \mu^{*}+\overline{\bar{\mu}}(\mu)+\tilde{\mu}(\mu)\right)\right\|=2\left\|f_{k}\left(x^{*}, \mu\right)\right\|
$$

since

$$
\| \tilde{\mu}(\mu)) \|=o\left(\sum_{k=1}^{p}\left\|f_{k}\left(x^{*}, \mu^{*}+\overline{\bar{\mu}}(\mu)\right)\right\|^{\frac{1}{k}}\right)
$$

where $\mu$ is sufficiently small.

Then, we substantiated that (3.3) holds. From this, it immediately yields that (3.2) is true for $\omega(\mu)=x(\mu)-x^{*}$ and $\|\omega(\mu)\|=o\left(\mu-\mu^{*}\right)$.

The last element of the proof, i.e., the continuity of $x(\mu)$, follows from the modified form of the Michael Selection Theorem 1.1.

Therefore, the multimapping $\Phi: C\left(V_{\varepsilon}(0)\right) \times V_{\varepsilon}(0) \rightarrow 2^{X \times M}$, which we defined in (3.4) by the formula

$$
\Phi(x, \tilde{\mu})=(x, \tilde{\mu})-\left\{\Psi_{p}(h)\right\}^{-1} F\left(x^{*}+x, \mu^{*}+t \bar{\mu}+\tilde{\mu}\right),
$$

gives us the continuity selector, i.e., we can choose the continuity solutions $(x(t \bar{\mu}), \tilde{\mu}(t \bar{\mu}))$ of $F$.

From the continuity of the function $x(t \bar{\mu})$, the continuity of the function $x(\mu)$ follows.

This finishes the proof of the Theorem.

Remark 3.3 If we assume that the spaces $X \times M$ and $Z$ are finite-dimensional, we can prove the existence of the continuous function $x(\mu)$ by the consideration of the following contraction process:

$$
\left(x_{k+1}, \tilde{\mu}_{k+1}\right)=\left(x_{k}, \tilde{\mu}_{k}\right)-\left\{\Psi_{p}(h)\right\}_{R}^{-1} F\left(x^{*}+x_{k}, \mu^{*}+t \bar{\mu}+\tilde{\mu}_{k}\right),
$$

where $\left\{\Psi_{p}(h)\right\}_{R}^{-1} z=\left(x_{z}, \mu_{z}\right)$ is the right inverse operator and

$$
\left\|\left(x_{z}, \mu_{z}\right)\right\|=\min _{\Psi_{p}(h)(x, \mu)=z}\|(x, \mu)\| .
$$

Such a process will converge to the continuity mapping $x(t \bar{\mu})$.

Analogously, we can prove the following two Theorems. 
Theorem 3.4 (The first $p$-order Implicit Function Theorem for the nontrivial kernel) Let $F(x, \mu) \in C^{p+1}(X \times M), F: X \times M \rightarrow Z$, where $M$ is finite-dimensional space, and $X, Z$ are Banach spaces. Assume that $F\left(x^{*}, \mu^{*}\right)=0$ and $\forall \bar{\mu} \in M,\|\bar{\mu}\|=1 ;(0, \bar{\mu}) \in$ $\bigcap_{k=1}^{p} \operatorname{Ker}^{k} f_{k}^{(k)}\left(x^{*}, \mu^{*}\right)$, that is,

$$
\left\|\left\{f_{1}^{\prime}\left(x^{*}, \mu^{*}\right)+f_{2}^{\prime \prime}\left(x^{*}, \mu^{*}\right)[0, \bar{\mu}]+\cdots+f_{p}^{(p)}\left(x^{*}, \mu^{*}\right)[0, \bar{\mu}]^{p-1}\right\}_{X}^{-1}\right\| \leq C .
$$

Then, there exists the mapping $x=x(\mu), \mu \in V_{\varepsilon}\left(\mu^{*}\right), x(\mu) \in C\left(V_{\varepsilon}\left(\mu^{*}\right)\right)$, for sufficiently small $\varepsilon>0$, such that $F(x(\mu), \mu)=0$ and

$$
\begin{aligned}
& x(\mu)=x^{*}+\omega(\mu), \quad\|\omega(\mu)\|=o\left(\left\|\mu-\mu^{*}\right\|\right), \\
& \left\|x(\mu)-x^{*}\right\| \leq C \sum_{k=1}^{p}\left\|f_{k}\left(x^{*}, \mu\right)\right\|_{Z_{k}}^{\frac{1}{k}}, \quad \forall \mu \in V_{\varepsilon}\left(\mu^{*}\right) .
\end{aligned}
$$

Theorem 3.5 (The second $p$-order Implicit Function Theorem for the nontrivial kernel) Let $F(x, \mu) \in C^{p+1}(X \times M), F: X \times M \rightarrow Z$, where $M$ is finite-dimensional space, and $X$ and $Z$ are Banach spaces. Let for $h_{\mu} \neq 0, h_{\mu} \in V_{\varepsilon}(0)$ there exist $\bar{h}_{x} \in X,\left\|\bar{h}_{x}\right\| \leq c<\infty$, such that $F$ is p-regular along $\bar{h}=\left[\bar{h}_{x}, \bar{h}_{\mu}\right]$, that is,

$$
\left\|\left\{f_{1}^{\prime}\left(x^{*}, \mu^{*}\right)+f_{2}^{\prime \prime}\left(x^{*}, \mu^{*}\right)[\bar{h}]+\cdots+\cdots+f_{p}^{(p)}\left(x^{*}, \mu^{*}\right)[\bar{h}]^{p-1}\right\}_{X}^{-1}\right\| \leq C,
$$

$\bar{h} \in \bigcap_{k=1}^{p} \operatorname{Ker}^{k} f_{k}^{(k)}\left(x^{*}, \mu^{*}\right), \bar{h}_{\mu}=\frac{h_{\mu}}{\left\|h_{\mu}\right\|}$.

Then, there exists the mapping $x=x(\mu), \mu \in V_{\varepsilon}\left(\mu^{*}\right), x(\mu) \in C\left(V_{\varepsilon}\left(\mu^{*}\right)\right)$, for sufficiently small $\varepsilon>0$, such that $F(x(\mu), \mu)=0$ and

$$
\begin{aligned}
& \mu=\mu^{*}+h_{\mu}, \quad x(\mu)=x^{*}+c(\mu) \bar{h}_{x}+\omega(\mu), \quad\|\omega(\mu)\|=o(\|\mu\|), c(\mu)=\|\mu\|, \\
& \left\|x(\mu)-x^{*}\right\| \leq C \sum_{k=1}^{p}\left\|f_{k}\left(x^{*}+h_{x}, \mu\right)\right\|_{Z_{k}}^{\frac{1}{k}} .
\end{aligned}
$$

\section{Application}

The illustration of our problem is the singular Van der Pol nonlinear boundary value problem

$$
F(x)=\ddot{x}+x+x^{p}=0, \quad x(0)=v, \quad x(2 \pi)=\rho,
$$

where $v$ and $\rho$ are small parameters from $U\left(v^{*}, \rho^{*}\right)=U(0,0), v^{*}=0, \rho^{*}=0$.

Note that (4.1) always has the trivial solution $x^{*}(t)=0$. We will consider the case $p=2$. We show that for any $h_{v}, h_{\rho}$ such that $h_{v} \neq h_{\rho}$, the mapping $F$ is 2-regular along element $H=\left[0, h_{v}, h_{\rho}\right]$, i.e., based on Theorem 3.1, there exists a continuous solution of (4.1) (with $p=2)$, dependent on parameters $\mu=(v, \rho)$, for $h_{v} \neq h_{\rho}$; for $h_{v}=h_{\rho}$, the mapping $F$ is 2-regular along element $H=\left[\sin t, h_{v}, h_{\rho}\right]$. All of this means that based on our Theorems, a continuous solution of Eq. (4.1), dependent on parameter $\mu$, exists for all sufficiently small $\mu$.

At the beginning, we note the following.

We take into account the equation

$$
\ddot{x}=f(x, v, \rho), \quad f: C^{2}[0,2 \pi] \times \mathbb{R}^{2} \rightarrow C[0,2 \pi], \quad x(0)=v, \quad x(2 \pi)=\rho,
$$


or

$$
F(x, v, \rho)=\ddot{x}-f(x, v, \rho)=0, \quad x(0)=v, \quad x(2 \pi)=\rho
$$

with boundary conditions, where $\mu \triangleq(v, \rho)$ and $v$ and $\rho$ are small parameters from $U\left(v^{*}, \rho^{*}\right)=U(0,0), v^{*}=0, \rho^{*}=0$.

In the case of a mapping $x(t, v, \rho)$ that is nonsingular at the solution point $x^{*}=x(t, 0,0)$ (in what follows, such types of mappings will be considered specifically), from the Implicit Function Theorem, it implies that for sufficiently small $(\nu, \rho)$, there exists $\Delta_{\nu}, \Delta_{\rho}$ and a function $x_{\mu}$ such that

$$
\begin{gathered}
x_{\mu}\left(0+\Delta_{\nu}\right)=0, x_{\mu}\left(2 \pi+\Delta_{\rho}\right)=0, \\
x_{\mu}(0)=v, \quad x_{\mu}(2 \pi)=\rho
\end{gathered}
$$

and

$$
c_{1}(|\nu|+|\rho|) \leq\left|\Delta_{\nu}\right|+|\Delta \rho| \leq c_{2}(|v|+|\rho|) .
$$

In this case, we can transfer the problem (4.2) to

$$
\ddot{x}=f(x, v, \rho), \quad x\left(\Delta_{v}\right)=0, \quad x\left(2 \pi+\Delta_{\rho}\right)=0
$$

and substitute

$$
t=\frac{1}{2 \pi}\left(2 \pi+\Delta_{\rho}-\Delta_{\nu}\right) \tau+\Delta_{\nu} .
$$

We obtain the problem (4.5) in the form

$$
\bar{F}\left(\bar{x}, \Delta_{v}, \Delta_{\rho}\right)=0,
$$

where $\bar{F}: X \rightarrow C[0,2 \pi], X=\left\{C^{2}[0,2 \pi] \times \mathbb{R}^{2} \mid \bar{x}(0)=0, \bar{x}(2 \pi)=0\right\}$ and

$$
\bar{x}(\tau)=x\left(\frac{1}{2 \pi}\left(2 \pi+\Delta_{\rho}-\Delta_{v}\right) \tau+\Delta_{v}\right) .
$$

We will call the mapping $\bar{F}$ the canonical form of the mapping $F$.

Example 4.1 Consider the following Van der Pol boundary value problem:

$$
F(x)=x^{\prime \prime}+x+x^{2}=0, \quad x(0)=v, \quad x(2 \pi)=\rho,
$$

where $v$ and $\rho$ are small parameters from $U\left(v^{*}, \rho^{*}\right)=U(0,0), v^{*}=0, \rho^{*}=0$. Substitute

$$
t=\frac{1}{2 \pi}\left(2 \pi+\Delta_{\rho}-\Delta_{\nu}\right) \tau+\Delta_{\nu} .
$$

We obtain the following equation:

$$
\frac{2 \pi}{2 \pi+\Delta_{\rho}-\Delta_{v}} \bar{x}^{\prime \prime}+\bar{x}+\bar{x}^{2}=0, \quad \bar{x}(0)=0, \quad \bar{x}(2 \pi)=0,
$$

where

$$
\bar{x}(\tau)=x\left(\frac{1}{2 \pi}\left(2 \pi+\Delta_{\rho}-\Delta_{v}\right) \tau+\Delta_{v}\right) .
$$

Taking this into account, we consider the following problem equivalent to (4.2):

$$
\frac{2 \pi}{2 \pi+\Delta_{\rho}-\Delta_{v}} \bar{x}^{\prime \prime}+\bar{x}+\bar{x}^{2}=0, \quad \bar{x}(0)=0, \quad \bar{x}(2 \pi)=0 .
$$


Let

$$
\bar{F}\left(\bar{x}(\tau), \Delta_{v}, \Delta \rho\right)=\bar{x}^{\prime \prime}+\left(1+\frac{\Delta_{\rho}}{2 \pi}-\frac{\Delta_{v}}{2 \pi}\right)\left(\bar{x}+\bar{x}^{2}\right)=0, \quad \bar{x}(0)=0, \bar{x}(2 \pi)=0,
$$

be the canonical form of Eq. (4.8). Then,

$$
\begin{aligned}
& \bar{F}^{\prime}\left(\bar{x}, \Delta_{v}, \Delta \rho\right)=\left[\frac{d^{2}}{d \tau^{2}}+\left(1+\frac{\Delta_{\rho}}{2 \pi}-\frac{\Delta_{v}}{2 \pi}\right)(1+2 \bar{x}),-\frac{1}{2 \pi}\left(\bar{x}+\bar{x}^{2}\right), \frac{1}{2 \pi}\left(\bar{x}+\bar{x}^{2}\right)\right], \\
& \bar{F}^{\prime}(0,0,0)=\left[\frac{d^{2}}{d \tau^{2}}+1,0,0\right] .
\end{aligned}
$$

We determine the kernel of the first derivative of the mapping $\bar{F}$ with respect to variable $\bar{x}$

$$
\operatorname{Ker} \bar{F}_{\bar{x}}^{\prime}(0,0,0)=\left\{\bar{x}: \bar{x}^{\prime \prime}+\bar{x}=0, \bar{x}(0)=\bar{x}(2 \pi)=0\right\} .
$$

From this, $\bar{x}(\tau)=c_{1} \cos \tau+c_{2} \sin \tau$. Taking into account the boundary conditions, we obtain $c_{1}=0, c_{2} \in \mathbb{R}$ and

$$
\operatorname{Ker} \bar{F}_{\bar{x}}^{\prime}(0,0,0)=\operatorname{span}\{\sin \tau\} .
$$

Let us evaluate the second derivative of the mapping $\bar{F}$

$$
\begin{aligned}
& \bar{F}^{\prime \prime}\left(\bar{x}, \Delta_{\nu}, \Delta_{\rho}\right)=\left[\begin{array}{ccc}
2\left(1+\frac{\Delta_{\rho}}{2 \pi}-\frac{\Delta_{v}}{2 \pi}\right) & -\frac{1}{2 \pi}(1+2 \bar{x}) & \frac{1}{2 \pi}(1+2 \bar{x}) \\
-\frac{1}{2 \pi}(1+2 \bar{x}) & 0 & 0 \\
\frac{1}{2 \pi}(1+2 \bar{x}) & 0 & 0
\end{array}\right], \\
& \bar{F}^{\prime \prime}(0,0,0)=\left[\begin{array}{ccc}
2 & -\frac{1}{2 \pi} & \frac{1}{2 \pi} \\
-\frac{1}{2 \pi} & 0 & 0 \\
\frac{1}{2 \pi} & 0 & 0
\end{array}\right] .
\end{aligned}
$$

Note that

$$
\begin{aligned}
& \bar{F}^{\prime \prime}(0,0,0)\left[h_{\bar{x}}, h_{v}, h_{\rho}\right]=\left[\begin{array}{ccc}
2 & -\frac{1}{2 \pi} & \frac{1}{2 \pi} \\
-\frac{1}{2 \pi} & 0 & 0 \\
\frac{1}{2 \pi} & 0 & 0
\end{array}\right]\left[\begin{array}{l}
h_{\bar{x}} \\
h_{v} \\
h_{\rho}
\end{array}\right]=\left[\begin{array}{c}
2 h_{\bar{x}}-\frac{1}{2 \pi} h_{v}+\frac{1}{2 \pi} h_{\rho} \\
-\frac{1}{2 \pi} h_{\bar{x}} \\
\frac{1}{2 \pi} h_{\bar{x}}
\end{array}\right], \\
& \bar{F}^{\prime \prime}(0,0,0)\left[h_{\bar{x}}, h_{\nu}, h_{\rho}\right]^{2}=2 h_{\bar{x}}^{2}-\frac{1}{\pi} h_{\bar{x}} h_{v}+\frac{1}{\pi} h_{\bar{x}} h_{\rho} .
\end{aligned}
$$

Introducing a scalar product in the form

$$
\forall z_{1}, z_{2} \in Z=C[0,2 \pi] \quad\left\langle z_{1}, z_{2}\right\rangle=\int_{0}^{2 \pi} z_{1}(\tau) z_{2}(\tau) d \tau,
$$

we describe the space $Z_{1}=\operatorname{Im} F_{\bar{x}}^{\prime}(0,0,0)$, such that $Z=Z_{1} \oplus W_{2}, W_{2}=\operatorname{Ker} F_{\bar{x}}^{\prime}(0,0,0)$. We have

$$
Z_{1}=\operatorname{Im} \bar{F}_{\bar{x}}^{\prime}(0,0,0)=\left\{z \in C[0,2 \pi]: \int_{0}^{2 \pi} z(\tau) \sin \tau d \tau\right\}
$$


It is easy to show that the projection onto $W_{2}$ has the form

$$
P_{W_{2}} z=\frac{1}{\pi} \sin t \int_{0}^{2 \pi} z(\tau) \sin \tau d \tau
$$

Therefore,

$$
\begin{aligned}
Z_{2} & =\operatorname{span}\left(\operatorname{Im} P_{W_{2}} \bar{F}^{\prime \prime}(0,0,0)[\cdot]^{2}\right) \\
& =\operatorname{span}\left\{z \in Z: z(t)=\frac{1}{\pi} \sin t \int_{0}^{2 \pi} \bar{F}^{\prime \prime}(0,0,0)[\cdot]^{2} \sin \tau d \tau\right\} \\
& =\operatorname{span}\{\sin t\}=W_{2},
\end{aligned}
$$

i.e., $P_{W_{2}}=P_{Z_{2}}$.

Now, we define the 2-factor operator

$$
\begin{aligned}
\forall \bar{h}= & {\left[h_{\bar{x}}, h_{v}, h_{\rho}\right] \in C^{2}[0,2 \pi] \times \mathbb{R} \times \mathbb{R} } \\
\Psi_{2}(h)[\bar{h}]= & \Psi_{2}\left((0,0,0),\left[h_{\bar{x}}, h_{\nu}, h_{\rho}\right]\right)\left[\bar{h}_{\bar{x}}, \bar{h}_{v}, \bar{h}_{\rho}\right] \\
= & \left(\bar{F}_{\bar{x}}^{\prime}(0,0,0)+P_{Z_{2}} \bar{F}^{\prime \prime}(0,0,0)\left[h_{\bar{x}}, h_{v}, h_{\rho}\right]\right)\left[\bar{h}_{\bar{x}}, \bar{h}_{\nu}, \bar{h}_{\rho}\right] \\
= & \frac{d^{2} h_{\bar{x}}}{d t^{2}}+h_{\bar{x}} \\
& +\frac{1}{\pi} \sin t \int_{0}^{2 \pi}\left(2 h_{\bar{x}} \bar{h}_{\bar{x}}-\frac{1}{2 \pi} h_{\nu} \bar{h}_{\bar{x}}+\frac{1}{2 \pi} h_{\rho} \bar{h}_{\bar{x}}-\frac{1}{2 \pi} h_{\bar{x}} \bar{h}_{\nu}+\frac{1}{2 \pi} h_{\bar{x}} \bar{h}_{\rho}\right) \sin \tau d \tau
\end{aligned}
$$

and 2-kernel of the 2-factor operator $\Psi_{2}(h)$

$$
\begin{aligned}
& \operatorname{Ker}^{2} \Psi_{2}(h)=\left\{h=\left[h_{\bar{x}}, h_{\nu}, h_{\rho}\right] \in C^{2}[0,2 \pi] \times \mathbb{R} \times \mathbb{R}:\right. \\
& \Psi_{2}\left((0,0,0),\left[h_{\bar{x}}, h_{\nu}, h_{\rho}\right]\right)\left[h_{\bar{x}}, h_{\nu}, h_{\rho}\right] \\
& \left.\frac{d^{2} h_{\bar{x}}}{d t^{2}}+h_{\bar{x}}+\frac{1}{\pi} \sin t \int_{0}^{2 \pi}\left(2 h_{\bar{x}}^{2}-\frac{1}{\pi} h_{\bar{x}} h_{v}+\frac{1}{\pi} h_{\bar{x}} h_{\rho}\right) \sin \tau d \tau=0\right\} .
\end{aligned}
$$

Taking into account the equations

$$
\int_{0}^{2 \pi} \sin ^{3} \tau d \tau=0, \quad \int_{0}^{2 \pi} \sin ^{2} \tau d \tau=\pi
$$

and the fact that $h_{\bar{x}}=q \sin \tau$ ( since $h_{\bar{x}} \in \operatorname{Ker} \bar{F}_{x}^{\prime}(0,0,0)$ ), we solve the following equation with unknown $q, h_{v}, h_{\rho}$ :

$$
\frac{1}{\pi} \sin t\left[q \int_{0}^{2 \pi}\left(\frac{1}{\pi} h_{\rho}-\frac{1}{\pi} h_{\nu}\right) \sin ^{2} \tau d \tau+2 q^{2} \int_{0}^{2 \pi} \sin ^{3} \tau d \tau\right]=0 .
$$

We obtain $\left(\frac{1}{\pi} h_{\rho}-\frac{1}{\pi} h_{v}\right) q=0$, i.e. $\left[q, h_{v}, h_{\rho}\right]=\left[0, h_{v}, h_{\rho}\right]$ or $\left[q, h_{v}, h_{\rho}\right]=\left[q, h_{\rho}, h_{\rho}\right]$, where $q, h_{\nu}, h_{\rho}$ are any constants belonging to $\mathbb{R}$.

From this,

$$
\operatorname{Ker}^{2} \Psi_{2}(h)=\left\{\left[0, h_{v}, h_{\rho}\right]\right\} \cup\left\{\left[q \sin t, h_{\rho}, h_{\rho}\right]\right\}, \quad q, h_{\nu}, h_{\rho} \in \mathbb{R}
$$

Now, we verify whether the 2 -factor operator is surjective onto $C[0,2 \pi]$ for elements $H$ belonging to the 2-kernel of the 2-factor operator.

Let $H=\left[q \sin t, h_{\rho}, h_{\rho}\right]$. We examine that

$$
\forall z \in C[0,2 \pi] \exists\left[\bar{h}_{\bar{x}}, \bar{h}_{v}, \bar{h}_{\rho}\right] \in C^{2}[0,2 \pi] \times \mathbb{R} \times \mathbb{R} \quad \Psi_{2}(H)\left[\bar{h}_{\bar{x}}, \bar{h}_{v}, \bar{h}_{\rho}\right]=z
$$


Using Lemma 2.14, it suffices to take any element $z=z_{2}=a \sin t \in Z_{2}=W_{2}=$ $\operatorname{Ker} \bar{F}_{\bar{x}}^{\prime}(0,0,0)=\operatorname{span}\{\sin t\}$. We are looking for an element $\bar{h}_{\bar{x}}=b \sin t \in \operatorname{Ker} \bar{F}_{\bar{x}}^{\prime}(0,0,0)$. After substitution to the obtained form of the 2-factor operator, we obtain

$$
\begin{aligned}
\frac{1}{\pi} & \sin t \int_{0}^{2 \pi}(q \sin \tau b \sin \tau \\
& \left.-\frac{1}{2 \pi} h_{\rho} b \sin \tau+\frac{1}{2 \pi} h_{\rho} b \sin \tau-\frac{1}{2 \pi} q \sin \tau \bar{h}_{v}+\frac{1}{2 \pi} q \sin \tau \bar{h}_{\rho}\right) \sin \tau d \tau \\
& =a \sin t
\end{aligned}
$$

From this, $\frac{1}{2 \pi} q\left(-\bar{h}_{v}+\bar{h}_{\rho}\right)=a$. Therefore, $\bar{h}_{\rho}=\bar{h}_{v}+\frac{2 \pi a}{q}$ and $\left[\bar{h}_{\bar{x}}, \bar{h}_{v}, \bar{h}_{\rho}\right]=$ $\left[b \sin t, \bar{h}_{v}, \bar{h}_{v}+\frac{2 \pi a}{q}\right]$ for any $b$. Therefore, $\Psi_{2}(H)$ is surjective, and this implies that the mapping $F$ is 2-regular at the point $(0,0,0)$ with respect to the element $H=\left[q \sin t, h_{\rho}, h_{\rho}\right]$.

Let $H=\left[0, h_{v}, h_{\rho}\right]$.

Using Lemma 2.14, we take any element $z=z_{2}=a \sin t \in Z_{2}=W_{2}=$ $\operatorname{Ker} \bar{F}_{\bar{x}}^{\prime}(0,0,0)=\operatorname{span}\{\sin t\}$. We look for element $\bar{h}_{\bar{x}}=b \sin t \in \operatorname{Ker} \bar{F}_{\bar{x}}^{\prime}(0,0,0)$. After substituting to the designated 2-factor operator's form, we obtain

$$
\frac{1}{\pi} \sin t \int_{0}^{2 \pi}\left(0-\frac{1}{2 \pi} h_{\nu} b \sin \tau+\frac{1}{2 \pi} h_{\rho} b \sin \tau-0+0\right) \sin \tau d \tau=a \sin t .
$$

Hence,

$$
\frac{1}{\pi} \sin t\left(-\frac{1}{2 \pi} h_{\nu} b \pi+\frac{1}{2 \pi} h_{\rho} b \pi\right)=a \sin t
$$

and

$$
b=\frac{2 \pi a}{h_{\rho}-h_{v}}
$$

for $h_{v} \neq h_{\rho}$.

Then, $\bar{F}$ is 2 -regular at the point $(0,0,0)$ with respect to element $H=\left[0, h_{v}, h_{\rho}\right]$, such that $h_{v} \neq h_{\rho}$. Therefore, based on our Theorem 3.1, we can formulate the following result for our Example 4.1.

Theorem 4.2 The mapping $\bar{F}(\bar{x}, \mu)$ is 2-regular at the point $(0,0,0)$ along $\bar{h}=\left(\bar{h}_{x}, \bar{h}_{\mu}\right)$, where $\bar{h}_{\mu}=\frac{h_{\mu}}{\left\|h_{\mu}\right\|}, h_{\mu}=(v, \rho)$ and

$$
\bar{h}_{x}(t, \mu)= \begin{cases}b \sin t, & b \neq 0, v=\rho, \\ 0, & v \neq \rho\end{cases}
$$

and for $\mu \in V_{\varepsilon}\left(\mu^{*}\right)$, for sufficiently small $\varepsilon>0$, there exists the continuous solution $x(t, \mu)$ of (4.8) such that

$$
x(t, \mu)=c(\mu) \sin t+\omega(t, \mu), \quad x(0, \mu)=v, \quad x(2 \pi, \mu)=\rho,
$$

where

$$
c(\mu)= \begin{cases}\left\|\mu-\mu^{*}\right\|, & v=\rho \\ o\left(\left\|\mu-\mu^{*}\right\|\right), & v \neq \rho\end{cases}
$$




\section{Conclusion}

By applying the $p$-regularity theory, we formulate and prove an analogue of the Lyusternik Theorem on the tangent cone, i.e., Theorem 3.1, which consists of the problem regarding the existence of continuous solutions of singular equations of the form $F\left(x^{*}, \mu^{*}\right)=0$, where $F(x, \mu) \in C^{p+1}(X \times M), F: X \times M \rightarrow Z, M$ is finite-dimensional space, and $X$ and $Z$ are Banach spaces. We illustrate this problem by a second-order Van der Pol differential Eq. (4.8) with boundary conditions.

Here, we provide the general result of our paper.

Consider the following differential equation:

$$
F(x, \mu)=F\left(x^{(k)}, \ldots, x^{\prime}, x, v, \rho\right)=0, \quad x(a)=v, \quad x(\mu)=\rho,
$$

where $F: C_{m}^{k}[a, b] \times \mathbb{R}^{m} \times \mathbb{R}^{m} \rightarrow C_{r}[a, b], x(t) \in C_{m}^{k}[a, b], v, \rho \in \mathbb{R}, \mu=(v, \rho)$. Analogously, (4.7) introduces $\bar{x}$ and the canonical map $\bar{F}(\bar{x}, \mu)$, where $\bar{F}: X \rightarrow C_{r}[a, b]$, $X=\left\{C_{m}^{k}[a, b] \times \mathbb{R}^{m} \times \mathbb{R}^{m} \mid \bar{x}(a)=0, \bar{x}(b)=0\right\}$.

Now, we can formulate the general result.

Theorem 5.1 Let $F(x, \mu) \in C^{p+1}\left(C_{m}^{k}[a, b] \times \mathbb{R}^{m} \times \mathbb{R}^{m}\right)$, and for any $h_{\mu} \neq 0, h_{\mu} \in$ $V_{\varepsilon}\left(\mu^{*}\right), h_{\mu}=(v, \rho)$, there exists $\bar{h}_{x}(t, \mu) \in X,\left\|\bar{h}_{x}(t, \mu)\right\| \leq c<\infty$ such that canonical mapping $\bar{F}(\bar{x}, \mu)$ is p-regular at $\left(\bar{x}^{*}, \mu^{*}\right)$ along $\bar{h}(\mu)=\left[\bar{h}_{x}(t, \mu), \bar{h}_{\mu}\right]$, where $\bar{h}(\mu) \in \bigcap_{k=1}^{p} \operatorname{Ker}^{k} \bar{f}_{k}^{(k)}\left(\bar{x}^{*}, \mu^{*}\right)$. Then, there exists the continuous mapping $x=x(t, \mu) \in$ $U_{\varepsilon}\left(x^{*}(t)\right), \mu \in V_{\varepsilon}\left(\mu^{*}\right), t \in[a, b], x(t, \mu) \in C\left(V_{\varepsilon}\left(\mu^{*}\right)\right)$, for sufficiently small $\varepsilon>0$, such that

$$
F(x(t, \mu), \mu)=0, \quad x(a)=v, \quad x(b)=\rho
$$

and

$$
x(t, \mu)=x^{*}+c(\mu) \bar{h}_{x}(t, \mu)+\omega(t, \mu),
$$

where

$$
c(\mu)=O\left(\left\|\mu-\mu^{*}\right\|\right), \quad \omega(t, \mu)=o\left(\left\|\mu-\mu^{*}\right\|\right) .
$$

Acknowledgements The results of the research of the first and second author carried out under the Research Theme No. 165/00/S were financed by the science grant granted by the Ministry of Science and Higher Education. The work of the second author was also supported by the Russian Foundation for Basic Research (Project Nos. 17-07-00510, 17-07-00493) and the RAS Presidium Programme (programme 27).

Open Access This article is licensed under a Creative Commons Attribution 4.0 International License, which permits use, sharing, adaptation, distribution and reproduction in any medium or format, as long as you give appropriate credit to the original author(s) and the source, provide a link to the Creative Commons licence, and indicate if changes were made. The images or other third party material in this article are included in the article's Creative Commons licence, unless indicated otherwise in a credit line to the material. If material is not included in the article's Creative Commons licence and your intended use is not permitted by statutory regulation or exceeds the permitted use, you will need to obtain permission directly from the copyright holder. To view a copy of this licence, visit http://creativecommons.org/licenses/by/4.0/.

\section{Appendix: Case $\sigma= \pm \sqrt{3}$}

Let us consider the boundary value problem

$$
\ddot{x}+\sigma\left(x^{2}-1\right) \dot{x}+x+x^{p}=0, \quad t \in[0,2 \pi] \quad x(0)=x(2 \pi)=0, \quad \sigma \in \mathbb{R}, \quad p \geq 2 .
$$


Consider the mapping

$$
F_{\sigma}: X \rightarrow Y, \quad F_{\sigma}(x)=\ddot{x}(\cdot)+\sigma\left(x^{2}(\cdot)-1\right) \dot{x}(\cdot)+x(\cdot)+x^{p}(\cdot)=0,
$$

where $X=\left\{x(\cdot) \in C^{p}[0,2 \pi]: x(0)=x(2 \pi)=0\right\}, Y=C[0,2 \pi]$.

Moreover

$$
F_{\sigma}^{\prime}(0) \xi=\ddot{\xi}(\cdot)-\sigma \dot{\xi}(\cdot)+\xi(\cdot), \quad \xi \in X .
$$

Consider the Sturm-Liouville problem

$$
\begin{aligned}
& \ddot{\xi}-\sigma \dot{\xi}+\xi=0, \\
& \xi(0)=\xi(2 \pi)=0 .
\end{aligned}
$$

This problem has finite number of eigenvalues pairs

$$
\sigma_{k}= \pm \sqrt{4-k^{2}}, \quad k=1,2
$$

and corresponding eigenfunctions

$$
h_{k}(t)=e^{\sigma_{k} \frac{t}{2}} \sin \left(\frac{t}{2} \sqrt{4-\sigma_{k}^{2}}\right), \quad k=1,2 .
$$

So we can show analogously the case $\sigma=0$ that the canonical mapping $\bar{F}_{\sigma}(\cdot)$ is 2-regular along elements $\bar{h}_{k}(\cdot)$ generated by $h_{k}(\cdot)$ (similary the case $\sigma=0$ ) for $k=1$ and $p=2$.

\section{References}

1. Brezhneva, O.A., Tret'yakov, A.A.: Implicit function theorems for nonregular mappings in Banach spaces. Exit from singularity. In: Banach Spaces and their Applications in Analysis, de Gruyter, pp. 285-302 (2007)

2. Brezhneva, O.A., Tret'yakov, A.A., Marsden, J.E.: Higher-order implicit function theorems and degenerate nonlinear boundary-value problems. Commun. Pure Appl. Anal. 7(2), 293-315 (2008)

3. Ioffe, A.D., Tihomirov, V.M.: Theory of Extremal Problems. North-Holland, Amsterdam (1979)

4. Izmailov, A.F., Tret'yakov, A.A.: Factor-Analysis of Nonlinear Mappings. Nauka, Moscow (1994). (in Russian)

5. Izmailov, A.F., Tret'yakov, A.A.: 2-Regular Solutions of Nonlinear Problems. Theory and Numerical Methods. Nauka, Moscow (1999). (in Russian)

6. Medak, B., Tret'yakov, A.A.: Existence of periodic solutions to nonlinear $p$-regular boundary value problem. Bound. Value Probl. 2015, 91 (2015). https://doi.org/10.1186/s13661-015-0360-2

7. Medak, B., Tret'yakov, A.A.: P-regularity Theory. Analysis and Applications. Fizmatlit, Moscow (2017). (in Russian)

8. Michael, E.A.: Continuous selector. Ann. Math. 64, 562-580 (1956)

9. Prusińska, A., Tret'yakov, A.A.: P-regularity theory. Tangent cone description in the singular case. Ukr. Math. J. 67(8), 1236-1246 (2016)

10. Tret'yakov, A.A.: The implicit function theorem in degenerate problems. Russ. Math. Surv. 42, 179-180 (1987)

11. Tret'yakov, A.A., Marsden, J.E.: Factor analysis of nonlinear mappings: p-regularity theory. Commun. Pure Appl. Anal. 2(4), 425-445 (2003)

Publisher's Note Springer Nature remains neutral with regard to jurisdictional claims in published maps and institutional affiliations. 